\title{
Estrutura da comunidade de invertebrados bentônicos em dois cursos d'água do Rio Grande do Sul, Brasil ${ }^{1}$
}

\author{
Alessandra A. P. Bueno ${ }^{2}$, Georgina Bond-Buckup ${ }^{2} \&$ Bibiana D. P. Ferreira ${ }^{2}$ \\ ${ }^{1}$ Contribuição número 400 do Departamento de Zoologia, Universidade Federal do Rio Grande. \\ 2 Departamento de Zoologia, Instituto de Biociências, PPG Biologia Animal, Universidade Federal do Rio Grande do Sul. \\ Avenida Bento Gonçalves 9500, prédio 43435, 91501-970 Porto Alegre, Rio Grande do Sul, Brasil. \\ E-mail: alebueno@ufrgs.br, ginabb@ufrgs.br
}

\begin{abstract}
Community structure of benthic invertebrates in two watercourses in Rio Grande do Sul State, southern Brazil. The benthic fauna has an important role in the trophic chain of limnic environments, serving as food for fishes and crustaceans. This work aimed to identify and compare, quantitative and qualitatively, the macrobenthic communities from two watercourses in Rio Grande do Sul State. Samplings were done with a Surber sampler, monthly, from September 1999 to August 2000, in one of the creeks forming Tainhas

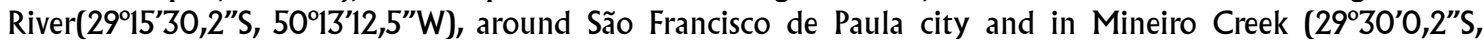
$50^{\circ} 46^{\prime} 50^{\prime \prime} \mathrm{W}$ ), around Taquara city. At each sampling point, physical and chemical variables of the waters were registered. In the laboratory, the samples were sorted out and the animals identified and quantified. Dissolved oxigen, $\mathrm{pH}$ and stream speed were very similar for both environments, whilst conductivity had extreme values. Insects, crustaceans, acari and molluscs dominated in the samples. Abundance, richness and diversity indexes in Tainhas subsidiary had relatively higher average values than Mineiro Creek. Similarity matrix groupings between sampling units indicate three groups. Our research revealed important characteristics of the ecology and distribution of benthic invertebrates, information that can subsidise future environmental monitoring in the region of São Francisco de Paula and Taquara.
\end{abstract}

KEY WORDS. Abiotic factors, benthic invertebrates, community structure, diversity indexes, Rio Grande do Sul.

A avaliação da dinâmica longitudinal das variáveis físicas e químicas dos rios pode fornecer subsídios para estudos que visam o melhor entendimento das características ecológicas de diversos grupos de organismos, principalmente os bentônicos, visto que as condições abióticas de um ambiente que freqüentemente determinam como os organismos que vivem em sistemas lóticos podem colonizar ou persistir em habitat novos ou em modificação (BRANCO \& NECCHI JR. 1997).

Os macroinvertebrados bentônicos constituem uma importante comunidade em rios, riachos e lagoas, servindo de alimento para peixes e crustáceos, e participando do fluxo de energia e da ciclagem de nutrientes. O seu uso como bioindicadores na qualidade das águas é igualmente recomendado, pois refletem as mudanças do ambiente (Esteves 1998). O conhecimento dessa fauna, portanto, constitui um passo fundamental para o entendimento das relações interespecíficas e do ecossistema como um todo.

A distribuição dos organismos aquáticos é o resultado da interação entre o hábito, condições físicas, que caracterizam o hábitat, e a disponibilidade alimentar (MerRIT \& Cummins 1984).
Dentre as variáveis abióticas de maior significância estão a velocidade da corrente, a temperatura e o oxigênio dissolvido na água. Em trechos de rios montanhosos a correnteza é considerada o fator que mais afeta a fauna. A velocidade da corrente, por sua vez, influencia o tamanho das partículas do substrato, afetando a distribuição do alimento e a remoção dos nutrientes. Por outro lado, a temperatura com variação em escala sazonal, diária e entre locais, é influenciada pelo clima, pela elevação e pela extensão da vegetação marginal (Allan 1995).

Alguns estudos têm investigado a estrutura da comunidade dos invertebrados bentônicos e as variáveis físicas e químicas dos rios brasileiros, destacando-se os trabalhos realizados no Estado de São Paulo por Uieda \& Gajardo (1996), BRANco \& Necchi Jr. (1997), KikUChi \& Uieda (1998), no Rio de Janeiro por BAPTISTA et al. (1998) e BAPTISTA et al. (2001a, b) e em Minas Gerais por MARques et al. (1999), CAllisto et al. (2001) e GALDEAN et al. (2001). Para as bacias hidrográficas do Rio Grande do Sul, no entanto, não há dados disponíveis até o momento.

Outras pesquisas foram desenvolvidas em diferentes países como aqueles em rios do Reino Unido por Wright et al. 
(1993), no sul da Nova Zelândia por Friberg et al (1997), na França por Beisel et al. (1998) e CHARVET et al. (1998), no Equador e Dinamarca por JaCoBsen et al. (1997) e JaCoBSEn \& ENCALADA (1998), em rios suíços por Lods-Crozet et al. (2001), nos Estados Unidos por Li et al. (2001), SPonseller et al. (2001), no Canadá por Rempel et al. (2000) e em rios da Noruega por Brittain et al. (2001), entre outros.

Esta pesquisa faz parte de um projeto que investiga a ecologia trófica de aeglídeos, que vem sendo desenvolvia no Laboratório de Carcinologia do Departamento de Zoologia da Universidade Federal do Rio Grande do Sul. A fauna bentônica dos rios onde estes crustáceos vivem ainda é desconhecida para a ciência. Portanto, este estudo tem como objetivo caracterizar a fauna bentônica presente em dois cursos d'água de altitudes diferentes no Rio Grande do Sul, avaliar suas densidades ao longo do ano e compará-las quanto à sua diversidade. Além disso, fornecer informações sobre quais grupos estão disponíveis como recurso alimentar para os crustáceos.

\section{MATERIAL E MÉTODOS}

\section{Área de Estudo}

A macrofauna bentônica foi coletada em um Arroio formador do Rio Tainhas, Bacia do Rio das Antas, no município de São Francisco de Paula $\left(29^{\circ} 15^{\prime} 30,2^{\prime \prime}\right.$ S e $\left.50^{\circ} 13^{\prime} 12,5^{\prime \prime} \mathrm{W}\right)$ e no Arroio do Mineiro, pertencente à Bacia do Rio Gravataí, município de Taquara $\left(29^{\circ} 30^{\prime} 0,2^{\prime \prime} \mathrm{S}\right.$ e $\left.50^{\circ} 46^{\prime} 50^{\prime \prime} \mathrm{W}\right)$. Para padronização da nomenclatura dos locais, o Arroio do Mineiro será citado em todo trabalho como Arroio de Taquara, devido ao fato do Arroio de Tainhas não possuir um registro específico.

A área amostrada em Tainhas, São Francisco de Paula, situa-se, a aproximadamente, $965 \mathrm{~m}$ acima do nível do mar e está localizada a $500 \mathrm{~m}$ da nascente do Rio Tainhas $\left(29^{\circ} 15^{\prime} 30,2^{\prime \prime} \mathrm{S}\right.$ e $\left.50^{\circ} 13^{\prime} 12,5^{\prime \prime} \mathrm{W}\right)$. Apresenta temperaturas média, máxima e mínima anuais do ar de $14,4^{\circ} \mathrm{C}, 20,3^{\circ} \mathrm{C}$ e $9,9^{\circ} \mathrm{C}$, respectivamente. Temperaturas negativas podem ocorrer nos meses de abril a novembro (IPAGRO 1989). A região possui altos níveis de pluviosidade em todos os meses do ano, sendo a precipitação média anual de $2252 \mathrm{~mm}$ (Nimer 1989). O arroio possui de 2 a $5 \mathrm{~m}$ de largura e profundidades variando entre 30 a $100 \mathrm{~cm}$. O substrato é predominantemente rochoso, com a presença desde seixos até areia fina, onde o fluxo da correnteza é menos intenso. Nas áreas onde a vegetação marginal cobre o arroio, observa-se um grande acúmulo de folhiço entre as pedras. A vegetação característica da região são pequenos arbustos pertencentes às mirtáceas e compostas, gramíneas altas e ciperáceas, com manchas esparsas do pinheiro brasileiro Araucaria angustifolia (Bert.) O. Ktze (Rambo 1956).

O município de Taquara está localizado na encosta nordeste do Estado do Rio Grande do Sul, sua altitude oscila entre 29 e $600 \mathrm{~m}$. Possui temperaturas média, máxima e mínima anuais do ar de $19,4^{\circ} \mathrm{C}, 26,8^{\circ} \mathrm{C}$ e $13,7^{\circ} \mathrm{C}$, respectivamente (IPAGro 1989). A precipitação média anual é de $1750 \mathrm{~mm}$, sendo julho, agosto e setembro os meses de máxima precipitação (NIMER 1989). O trecho estudado do Arroio do Mineiro está a $300 \mathrm{~m}$ de altitude $\left(29^{\circ} 30^{\prime} 0,2^{\prime \prime} \mathrm{S}\right.$ e $\left.50^{\circ} 46^{\prime} 50^{\prime \prime} \mathrm{W}\right)$, possui de 4 a $5 \mathrm{~m}$ de largura e profundidades variando entre 30 e $60 \mathrm{~cm}$. O substrato é semelhante ao Arroio de Tainhas, porém com predominância de cascalho e areia mais fina. A vegetação marginal é modificada pela influência antrópica, com a presença de estradas e plantações de hortaliças.

Revista Brasileira de Zoologia 20 (1): 115-125, março 2003

\section{Amostragens}

As amostragens apoiaram-se nas metodologias mencionadas por Southwood (1978) e MerRitT \& Cummins (1984), utilizando-se um amostrador tipo Surber $(33 \times 33 \mathrm{~cm})$ com malha de $250 \mu \mathrm{m}$. Nos dois arroios, o trecho amostrado de $60 \mathrm{~m}$ foi demarcado com 31 estacas, com dois metros de distância uma da outra. Uma linha imaginária foi traçada ao longo do arroio, dividindo-os em lado direito e esquerdo. Foram determinados 124 pontos, sendo que os ímpares ficaram do lado esquerdo e os pares do lado direito. Mensalmente, de setembro/99 a agosto/00, foram sorteados quatro pontos de amostragem em cada arroio. No ponto sorteado, colocou-se o amostrador contra a correnteza e durante um minuto o substrato foi remexido. $\mathrm{O}$ material coletado foi então fixado em etanol a $70 \%$.

No local de coleta, foram registradas as seguintes variáveis físicas e químicas dos ambientes: a) a velocidade da corrente, verificada através do método de objetos impulsionados pela correnteza (SchWoerbel 1975); b) a temperatura da água, medida com termômetro de escala interna; c) o oxigênio dissolvido, medido com auxílio de um termo-oxímetro portátil OXI 330/ SET-WTW; d) o pH, registrado com auxílio de um medidor portátil Cole \& Parmer; e) a condutividade elétrica, medida com condutivímetro portátil YST Model 33.

Amostras da água foram coletadas nos meses de outubro/ 99, maio/00 e agosto/00 no Arroio de Taquara e nos meses de agosto/99 e agosto/00 no Arroio de Tainhas para análise dos seguintes nutrientes: nitrogênio, fósforo, potássio, cálcio, magnésio, cobre, zinco, ferro, manganês, sódio, boro e enxofre total, nitrito+nitrato e o íon amônio.

No laboratório os organismos foram triados, quantificados e identificados segundo Edmondson (1959), Borror \& DeLong (1969), Macan (1975) e Pérez (1988).

\section{Ánalise de dados}

Para melhor interpretação e comparação dos resultados, os dados foram agrupados por estação do ano (Primavera = setembro, outubro e novembro; Verão = dezembro e janeiro; Outono = março, abril e maio; Inverno = junho, julho e agosto). No mês de fevereiro não foi possível realizar as coletas.

As análises estatísticas foram realizadas pelo pacote estatístico SPSS (versão10.0) e os índices de diversidade calculados segundo Poole (1974), Magurran (1988) e Krebs (1989).

A diversidade da comunidade dos dois arroios foi estimada pelo índice de Shannon-Wiener $\left(\mathrm{H}^{\prime}\right)$, que valoriza a abundância proporcional das espécies enfatizando a riqueza e homogeneidade. Também utilizou-se o índice de Margalef $\left(\mathrm{D}_{\mathrm{Mg}}\right)$, que expressa a riqueza ponderada pelo tamanho amostral e pelo recíproco de Simpson (1/D), que representa a medida de dominância influenciada pelas espécies mais comuns, ou seja, probabilidade de dois indivíduos capturados ao acaso pertencerem a mesma espécie.

Foram calculados, também, a riqueza de espécies (S), a Equitatividade (E), calculada com base no $\mathrm{H}^{\prime}$ e na abundância das espécies na comunidade. A riqueza de EPT (Ephemeroptera+ Plecoptera + Trichoptera), também foi calculada por estas ordens serem consideradas, por vários autores, como indicadoras de qualidade da água (CAllisto \& Esteves 1998).

Na comparação da diversidade dos dois arroios foi utilizado o índice de similaridade de Jaccard (J), além do Teste $t$ de Student que compara os $\mathrm{H}^{\prime}$ dos arroios. 
Os dados da fauna, por ponto de amostragem, foram agrupados por mês, sendo que o mês equivale a uma unidade amostral. Para detecção de padrões de distribuição dos organismos e suas relações com as variáveis ambientais os dados foram submetidos à análise multivariada utilizando-se o aplicativo MULTIV (PILLAR 1997). O programa e o manual estão divulgado eletronicamente no site (http: $\backslash \backslash$ ecoqua.ecologia. ufrgs.br). As análises descritas a seguir foram utilizadas para verificar a significância de cada teste realizado pelo aplicativo. A correlação entre a abundância dos organismos e as variáveis abióticas foi testada por meio do teste de Mantel. Foram executadas análises multivariadas de ordenação e agrupamento e determinadas as medidas de congruência (MANTEL 1967). Foram conduzidos testes de significância de eixos de ordenação (PIllar 1999a), nitidez de grupos nas análises de agrupamentos detectados a partir de um teste baseado em auto-reamostragem (bootstrap) com 1000 iterações usando um limiar de probabilidade $(\alpha)$ de 0,1 (Pillar 1999b) e testes de aleatorização (Pillar \& Orloci 1996). Essas análises foram baseadas em medidas de dissimilaridade (Distância Euclidiana) entre cada par de unidade amostral.

Para um melhor ajuste dos dados, as espécies com menos de oito ocorrências nos meses de amostragem foram eliminadas da análise.

$\mathrm{Na}$ análise de agrupamento das unidades amostrais utilizou-se o critério da variância mínima (soma dos quadrados), a partir da Distância Euclidiana, sendo os dados transformados em raiz quadrada. Na ordenação das unidades amostrais foi empregada a Análise de Coordenadas Principais (PCoA), aplicada à matriz de semelhança já descrita.

\section{RESULTADOS}

\section{Variáveis Abióticas}

A porcentagem de saturação do oxigênio dissolvido mostrou-se semelhante nos dois locais, porém com variação entre as estações do ano. Valores mais altos foram observados no inverno, em Tainhas, e na primavera, em Taquara (Fig. 1). $\mathrm{O} \mathrm{pH}$ revelou valores neutros ao longo do ano em ambos arroios, porém o Arroio de Tainhas apresentou uma grande oscilação na primavera, com valores entre 6,5 e 9,0 (Fig. 2). A velocidade da correnteza também variou bastante na primavera, em Tainhas, e a estação com maior fluxo, em Taquara, foi no inverno (Fig. 3). Entretanto, observou-se valores de condutividade bastante distintos, porém constantes nas estações do ano em ambos os arroios. Em Tainhas, a condutividade esteve em torno de $20 \mathrm{mS} / \mathrm{cm}$ e em Taquara, os valores foram sempre acima de $60 \mathrm{mS} / \mathrm{cm}$ (Fig. 4). A figura 5 apresenta os valores máximos, mínimos e médios da temperatura da água nos locais de coleta, na qual observa-se que o Arroio de Taquara possui temperaturas mais elevadas em todas as estações do ano, exceto no inverno.

A análise química da água revelou maiores concentrações dos nutrientes fósforo, cálcio, magnésio e sódio nas amostras de Taquara, enquanto que os demais elementos analisados apresentaram valores semelhantes em ambos arroios (Tab. I).

\section{Comunidade de Invertebrados Bentônicos}

Durante o período amostrado foram obtidos 27.963 indivíduos, sendo que 16.059 em Tainhas e 11.904 em Taquara, representados por 10 ordens e 40 famílias de Insecta, além de
Tabela I. Concentrações dos nutrientes analisados nos Arroios de Tainhas e Taquara, Rio Grande do Sul.

\begin{tabular}{lccccccc}
\hline \multirow{2}{*}{ Nutrientes (mg/L) $)$} & \multicolumn{2}{c}{ Tainhas } & & \multicolumn{3}{c}{ Taquara } \\
\cline { 2 - 3 } \cline { 6 - 7 } & Ago/1999 & Ago/2000 & & Out/1999 & Mai/2000 & Ago/2000 \\
\hline Nitrogênio total & $*$ & $<0,1$ & & 0,4 & $<0,1$ & $<0,1$ \\
Fósforo total & $<0,01$ & 0,01 & & 0,09 & 0,06 & 0,04 \\
Potássio total & 1,3 & 1,4 & & 1,7 & 2,6 & 2,0 \\
Cálcio total & 1,2 & 0,73 & & 6,3 & 7,5 & 6,6 \\
Magnésio total & 0,64 & 0,47 & & 3,7 & 3,6 & 3,4 \\
Cobre total & $<0,01$ & $<0,02$ & $<0,01$ & $<0,02$ & $<0,02$ \\
Zinco total & 0,01 & 0,01 & $<0,01$ & $<0,01$ & $<0,01$ \\
Ferro total & 0,16 & 0,2 & & 0,65 & 0,63 & 1,5 \\
Manganês total & $<0,01$ & 0,01 & 0,03 & 0,12 & 0,03 \\
Sódio total & 1,7 & 2,2 & & 5,1 & 7,0 & 6,4 \\
Boro total & 0,12 & 0,04 & 0,28 & 0,03 & $<0,01$ \\
Enxofre total & $<0,08$ & $*$ & 1,7 & $*$ & $*$ \\
Nitrito+nitrato & $<0,04$ & $*$ & 0,3 & $<0,1$ & $*$ \\
Ín amônio & $<0,04$ & $*$ & $<0,1$ & 0,2 & $*$ \\
\hline
\end{tabular}

* Nutrientes não analisados.

Crustacea, Acarina e Mollusca, entre outros. A freqüência absoluta e relativa dos organismos mais abundantes coletados por estação do ano e por local, durante o período estudado, está representada na tabela II. A maior parte das famílias de Insecta identificadas foram encontradas nos dois ambientes, entretanto, algumas famílias estiveram presentes somente em um dois arroios. Foram encontrados, apenas em Tainhas, exemplares de Euthyplocidae (Ephemeroptera) e Naucoridae (Hemiptera). Em Taquara, os organismos exclusivos deste ambiente foram Ceratopogonidae (Diptera), Hypogastruridae (Collembola), Pyraliade (Lepidoptera), Hedridae e Gerridae (Hemiptera).

As famílias Chironomidae e Simullidae (Diptera) foram mais abundantes, durante a primavera e verão, no Arroio de Taquara, já no outono e inverno os Chironomidae foram mais freqüentes em Tainhas. Leptophlebiidae (Ephemeroptera), família indicadora de águas com grande quantidade de matéria orgânica (Perez 1988), foi mais abundante em Taquara na primavera e no verão e em Tainhas no outono e inverno. Com relação às famílias de Coleoptera, Elmidae predominou em Taquara (primavera/verão), enquanto que Psephenidae foi mais abundante em Tainhas. Em Tainhas, também, foram mais abundantes os ácaros e os microcrustáceos representados pelos Cladocera e Copepoda.

Comparando-se os arroios, Tainhas apresentou abundância média maior no outono e inverno (Fig. 6). A riqueza e o índice de Shannon $\left(\mathrm{H}^{\prime}\right)$, para o mesmo arroio, mostraram valores médios mais elevados em todas as estações do ano, exceto no verão (Figs 7 e 8). O índice de Margalef e Recíproco de Simp- 

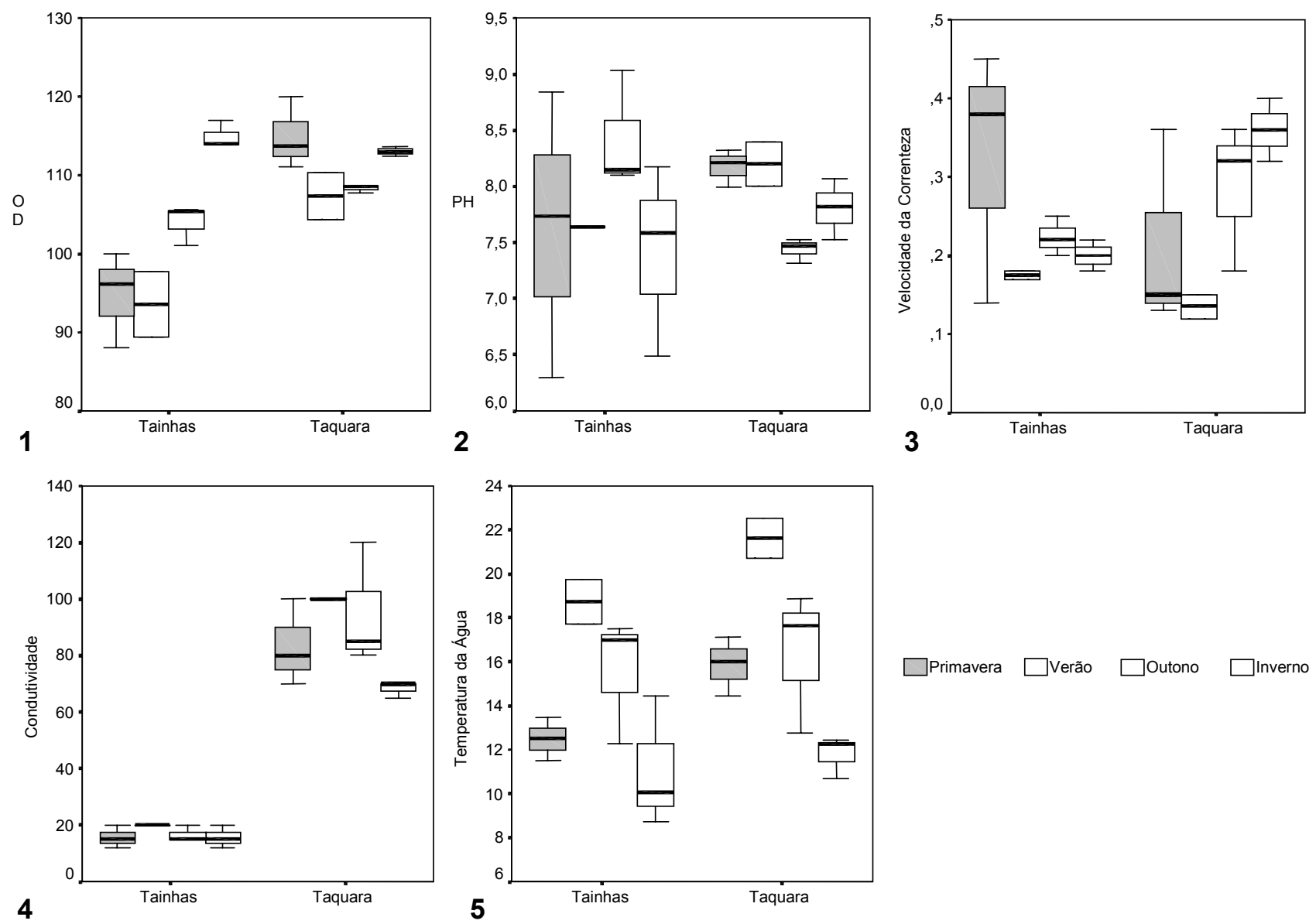

Figuras 1-5. Valores mínimos, máximos e médios de variáveis físicas e químicas para os Arroios Tainhas e Taquara, Rio Grande do Sul, durante as estações do ano, no período de setembro/1999 a agosto/2000. (1) OD saturação do oxigênio dissolvido (\%); (2) pH; (3) velocidade da correnteza $(\mathrm{m} / \mathrm{s}) ;(4)$ condutividade $(\mathrm{mS} / \mathrm{cm}) ;(5)$ temperatura da água $\left({ }^{\circ} \mathrm{C}\right)$. Caixa = média \pm desvio padrão, linha $=$ valores mínimos e máximos.

son, também, foram mais elevados em Tainhas do que em Taquara, com exceção do verão (Figs 9 e 10). A equitatividade, entretanto, foi maior na primavera e verão no Arroio de Tainhas e no outono e inverno no Arroio de Taquara (Fig. 11). Observase, também, que o inverno foi a estação com valores máximos mais elevados para o Arroio de Tainhas, com exceção da abundância, enquanto que, em Taquara houve uma variação nos índices ao longo das estações. A abundância e riqueza tiveram valores máximos no verão, o $\mathrm{H}^{\prime}$ e a Equitatividade no outono e o índice de Margalef e Recíproco de Simpson no inverno. Os valores de riqueza de EPT (Ephemeroptera + Plecoptera + Trichoptera) foram maiores em Tainhas em todas as estações do ano (Fig. 12).

Os organismos foram agrupados em coletores, filtradores, predadores e raspadores segundo a classificação de grupos funcionais de alimentação de MERRIT \& Cummins (1984). Não houve diferença significatica entre os arroios, porém, todos os grupos foram mais freqüentes em Tainhas. Observa-se, ainda, que os coletores foram os organismos mais abundantes em ambos os arroios (Fig. 13).

Os dois ambientes diferem com relação ao número de espécies, verificado por meio do índice de similaridade de Jaccard. Os valores mensais variam de 0,47 a 0,66 , sendo que, o menor índice foi no mês de setembro e o maior em julho (Fig. 14). O teste $t$ realizado entre o $\mathrm{H}^{\prime}$ de Taquara e o $\mathrm{H}^{\prime}$ de Tainhas, calculado para todo o período estudado, revelou que houve diferença significativa entre os dois locais (tcalc: 2,$78 ; \mathrm{gl}: 23.676 ; \alpha$ : 0,01 ).

Os resultados da análise de agrupamento, da matriz de similaridade entre as unidades amostrais, indicaram a formação de três grupos distintos (Fig. 15 e Tab. III). Os grupos formados no dendograma mostraram a separação da fauna dos arroios. O grupo 1 representa as unidades amostrais de Tainhas, o grupo 2 as amostras dos meses de novembro, dezembro e janeiro de Taquara e o terceiro grupo é formado pelos demais meses de Taquara e pelos meses de setembro e outubro de Tainhas.

O diagrama de dispersão apresentado na figura 16 expressa a variação das 22 unidades amostrais ao longo do ano. Observa-se, novamente, a separação entre os locais de coleta. Os pon-

Revista Brasileira de Zoologia 20 (1): 115-125, março 2003 

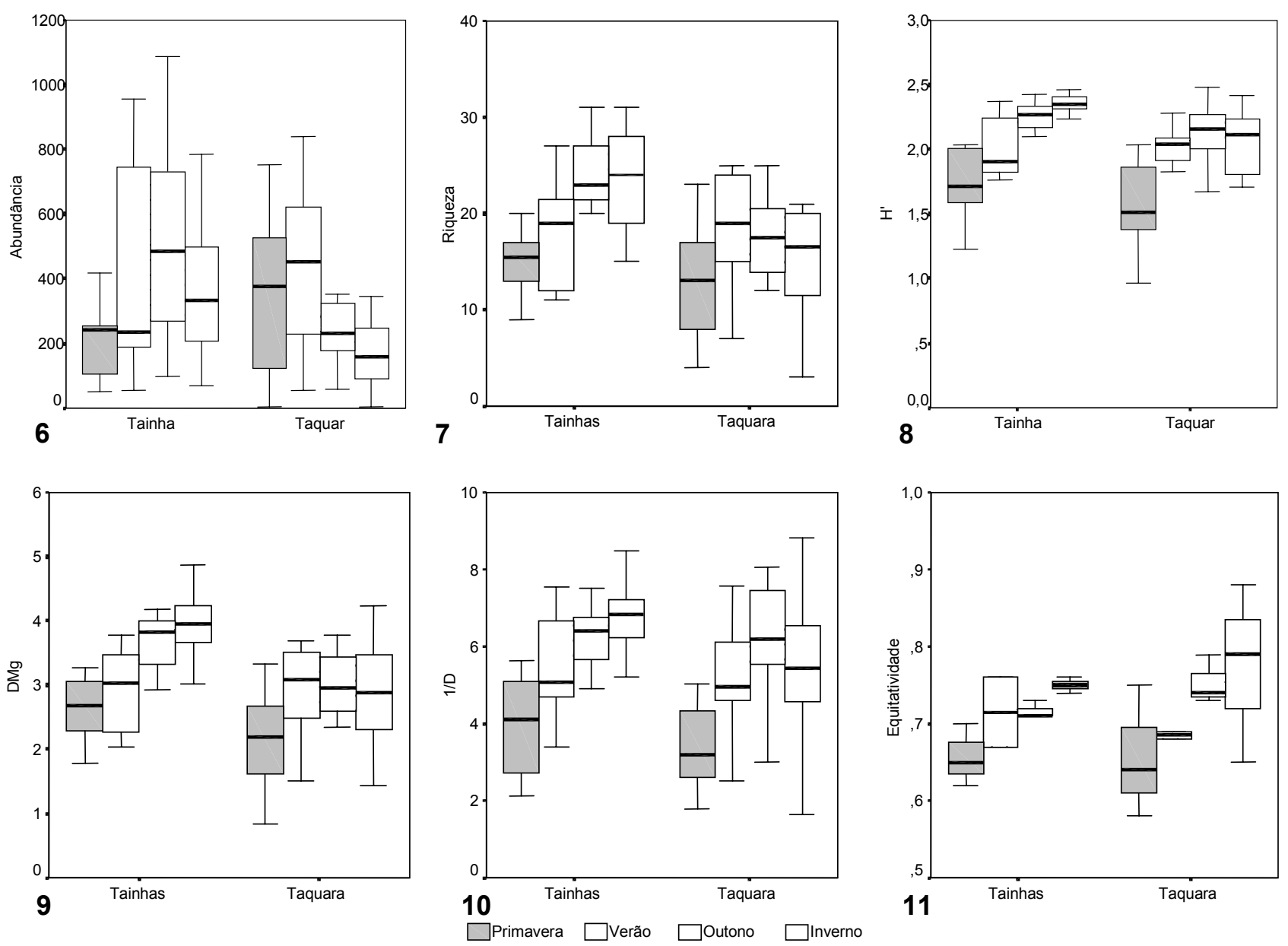

Figuras 6-11. Valores mínimos, máximos e médios da macrofauna bentônica calculados para os Arroios Tainhas e Taquara, Rio Grande do Sul, durante as estações do ano, no período de setembro/1999 a agosto/2000. (6) Abundância; (7) riqueza; (8) Índice de ShannonWiener $\left(H^{\prime}\right)$; (9) Índice de Margalef $\left(D_{M g}\right)$; (10) Recíproco de Simpsom (1/D); (11) equitatividade. Caixa = média \pm desvio padrão, linha = valores mínimos e máximos.

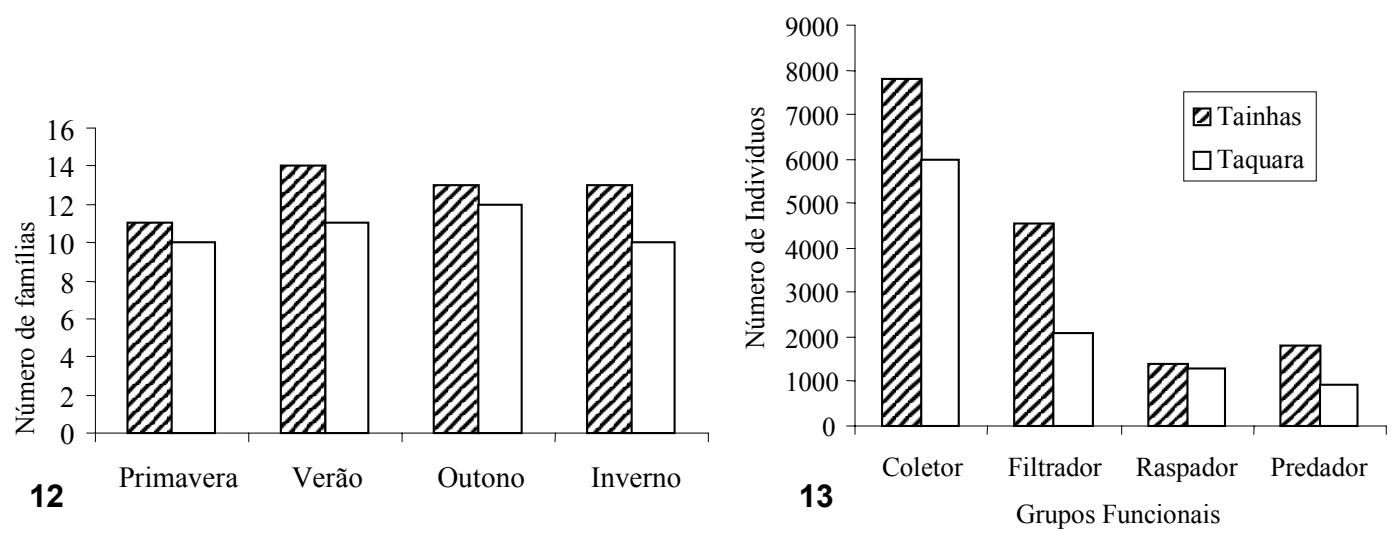

Figuras 12-13. (12) Número de famílias de EPT (Ephemeroptera + Plecoptera + Trichoptera) nas estações do ano e (13), número de indivíduos da macrofauna bentônica presentes nos grupos funcionais dos Arroios de Tainhas e Taquara, Rio Grande do Sul, durante o período de setembro/1999 a agosto/2000. 
Tabela II. Freqüências absoluta, relativa (\%) e grupos funcionais dos táxons mais abundantes da fauna coletada nos Arroio de Tainhas e Taquara, Rio Grande do Sul, durante as estações do ano, no período de setembro/99 a agosto/00.

\begin{tabular}{|c|c|c|c|c|c|c|c|c|c|}
\hline & \multicolumn{2}{|c|}{ Primavera } & \multicolumn{2}{|c|}{ Verão } & \multicolumn{2}{|c|}{ Outono } & \multicolumn{2}{|c|}{ Inverno } & \multirow{2}{*}{$\begin{array}{c}\text { Grupo } \\
\text { funcional }\end{array}$} \\
\hline & Tainhas & Taquara & Tainhas & Taquara & Tainhas & Taquara & Tainhas & Taquara & \\
\hline Nematoda & $0(0,00)$ & $9(0,29)$ & $10(0,32)$ & $14(0,43)$ & $3(0,04)$ & $8(0,28)$ & $21(0,49)$ & $9(0,46)$ & coletor \\
\hline \multicolumn{10}{|l|}{ Annelida } \\
\hline Oligochaeta & $168(8,08)$ & $191(6,19)$ & $597(19,32)$ & $121(3,77)$ & $615(10,21)$ & $107(3,85)$ & $198(4,64)$ & $304(15,58)$ & filtrador \\
\hline Mollusca & $4(0,19)$ & $36(1,16)$ & $7(0,22)$ & $51(1,59)$ & $30(0,49)$ & $35(1,25)$ & $8(0,18)$ & $19(0,97)$ & raspador \\
\hline Acarina & $92(4,42)$ & $65(2,10)$ & $331(10,71)$ & $151(4,71)$ & $496(8,24)$ & $147(5,29)$ & $411(9,65)$ & $102(5,22)$ & predador \\
\hline \multicolumn{10}{|l|}{ Diptera } \\
\hline Chironomidae & $911(43,86)$ & $1508(48,92)$ & $992(32,10)$ & $1095(34,18)$ & $1870(31,06)$ & $633(22,78)$ & $1308(30,71)$ & $434(22,24)$ & coletor \\
\hline Simuliidae & $48(2,31)$ & $533(17,29)$ & $3(0,09)$ & $182(5,68)$ & $38(0,63)$ & $149(5,36)$ & $27(0,63)$ & $115(5,89)$ & filtrador \\
\hline \multicolumn{10}{|l|}{ Ephemeroptera } \\
\hline Leptophlebiidae & $154(7,41)$ & $245(7,94)$ & $200(6,47)$ & $257(8,02)$ & $527(8,75)$ & $474(17,06)$ & $565(13,26)$ & $252(12,91)$ & coletor \\
\hline Baetidae & $82(3,94)$ & $162(5,25)$ & $231(7,47)$ & $549(17,14)$ & $447(7,42)$ & $323(11,62)$ & $205(4,81)$ & $107(5,48)$ & raspador \\
\hline Caenidae & $21(1,01)$ & $9(0,29)$ & $18(0,58)$ & $381(11,89)$ & $238(3,95)$ & $14(0,50)$ & $195(4,57)$ & $47(2,40)$ & coletor \\
\hline Euthyplociidae & $11(0,53)$ & $0(0,00)$ & $1(0,03)$ & $0(0,00)$ & $71(1,17)$ & $0(0,00)$ & $57(1,33)$ & $0(0,00)$ & raspador \\
\hline \multicolumn{10}{|l|}{ Trichoptera } \\
\hline Hydropsychidae & $1(0,04)$ & $48(1,55)$ & $51(1,65)$ & $56(1,74)$ & $118(1,96)$ & $43(1,54)$ & $134(3,14)$ & $26(1,33)$ & filtrador \\
\hline Hydrobiosidae & $29(1,39)$ & $26(0,84)$ & $11(0,35)$ & $18(0,56)$ & $12(0,19)$ & $38(1,36)$ & $12(0,28)$ & $2(0,10)$ & predador \\
\hline Leptoceridae & $44(2,11)$ & $5(0,16)$ & $36(1,16)$ & $26(0,81)$ & $63(1,04)$ & $2(0,07)$ & $60(1,40)$ & $4(0,20)$ & coletor \\
\hline Philopotamidae & $0(0,00)$ & $0(0,00)$ & $23(0,74)$ & $4(0,12)$ & $256(4,25)$ & $28(1,00)$ & $90(2,11)$ & $10(0,51)$ & filtrador \\
\hline \multicolumn{10}{|l|}{ Plecoptera } \\
\hline Gripopterygidae & $7(0,33)$ & $15(0,48)$ & $25(0,80)$ & $1(0,03)$ & $64(1,06)$ & $33(1,18)$ & $131(3,07)$ & $67(3,43)$ & predador \\
\hline Perlidae & $10(0,48)$ & $5(0,16)$ & $20(0,64)$ & $20(0,62)$ & $21(0,34)$ & $104(3,74)$ & $26(0,61)$ & $23(1,17)$ & predador \\
\hline \multicolumn{10}{|l|}{ Coleoptera } \\
\hline Elmidae & $74(3,56)$ & $115(3,73)$ & $72(2,33)$ & $144(4,49)$ & $200(3,32)$ & $174(6,26)$ & $122(2,86)$ & $83(4,25)$ & coletor \\
\hline Psephenidae & $31(1,49)$ & $0(0,00)$ & $52(1,68)$ & $8(0,24)$ & $94(1,56)$ & $2(0,07)$ & $59(1,38)$ & $0(0,00)$ & raspador \\
\hline \multicolumn{10}{|l|}{ Odonata } \\
\hline Coenagrionidae & $2(0,09)$ & $14(0,45)$ & $5(0,16)$ & $0(0,00)$ & $22(0,36)$ & $47(1,69)$ & $15(0,35)$ & $6(0,30)$ & predador \\
\hline \multicolumn{10}{|l|}{ Hemiptera } \\
\hline Naucoridae & $5(0,24)$ & $0(0,00)$ & $4(0,12)$ & $0(0,00)$ & $17(0,28)$ & $0(0,00)$ & $12(0,28)$ & $0(0,00)$ & predador \\
\hline \multicolumn{10}{|l|}{ Crustacea } \\
\hline Amphipoda & $0(0,00)$ & $11(0,35)$ & $0(0,00)$ & $11(0,34)$ & $0(0,00)$ & $6(0,21)$ & $3(0,07)$ & $13(0,66)$ & coletor \\
\hline Copepoda & $284(13,67)$ & $30(0,97)$ & $310(10,03)$ & $106(3,30)$ & $700(11,62)$ & $242(8,71)$ & $471(11,05)$ & $257(13,17)$ & filtrador \\
\hline Cladocera & $87(4,18)$ & $0(0,00)$ & $80(2,58)$ & $2(0,06)$ & $11(0,18)$ & $150(5,39)$ & $27(0,63)$ & $35(1,79)$ & filtrador \\
\hline Ostracoda & $12(0,57)$ & $3(0,09)$ & $10(0,32)$ & $4(0,12)$ & $99(1,64)$ & $17(0,61)$ & $98(2,30)$ & $27(1,38)$ & filtrador \\
\hline Aeglidae & $10(0,48)$ & $52(1,68)$ & $1(0,03)$ & $2(0,06)$ & $7(0,11)$ & $2(0,07)$ & $4(0,09)$ & $9(0,46)$ & predador \\
\hline Total & $2087(100)$ & $3082(100)$ & $3090(100)$ & $3203(100)$ & $6019(100)$ & $2778(100)$ & $4259(100)$ & $1951(100)$ & \\
\hline
\end{tabular}

Revista Brasileira de Zoologia 20 (1): 115-125, março 2003 


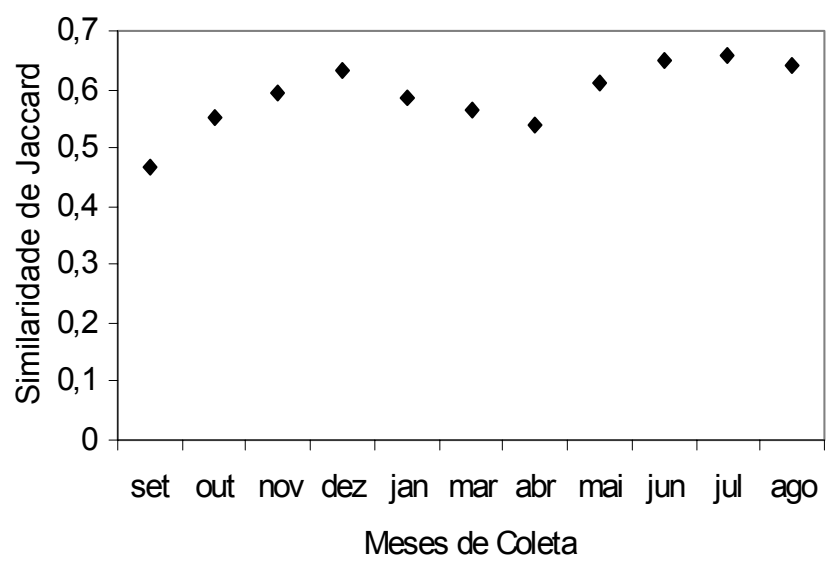

Figura 14. Variação mensal dos índices de similaridade de Jaccard a macrofauna bentônica dos Arroios de Tainhas e Taquara, Rio Grande do Sul, obtidos durante o período de setembro/1999 a agosto/2000.

Tabela III. Valores de ('bootstrap') gerados em 1000 iterações, obtidos no teste de nitidez de grupos na Análise de Agrupamento da figura 10. (N) Tamanho das amostras em 16 passos de amostragem; valores significativos: $p \geq 0,1$.

\begin{tabular}{cccc}
\hline$N$ & Dois grupos & Três grupos & Quatro grupos \\
\hline 7 & 0,205 & 0,119 & 0,058 \\
8 & 0,195 & 0,134 & 0,054 \\
9 & 0,207 & 0,119 & 0,046 \\
10 & 0,192 & 0,115 & 0,044 \\
11 & 0,190 & 0,111 & 0,033 \\
12 & 0,179 & 0,110 & 0,040 \\
13 & 0,168 & 0,098 & 0,046 \\
14 & 0,159 & 0,111 & 0,031 \\
15 & 0,179 & 0,141 & 0,037 \\
16 & 0,192 & 0,100 & 0,037 \\
17 & 0,185 & 0,119 & 0,036 \\
18 & 0,178 & 0,113 & 0,032 \\
19 & 0,187 & 0,103 & 0,032 \\
20 & 0,192 & 0,124 & 0,033 \\
21 & 0,198 & 0,105 & 0,040 \\
22 & 0,178 & 0,114 & 0,047 \\
\hline
\end{tabular}

tos que representam Tainhas encontram-se localizados embaixo e à esquerda, associados aos meses de novembro a agosto, enquanto que os pontos relacionados ao Taquara encontram-se embaixo e em cima, à direita. Entretanto, nota-se que os pontos 1 e 2 , que representam os meses de setembro e outubro de Tainhas, encontram-se misturados aos de Taquara. Estes pontos são os mais semelhantes quanto ao número de famílias e abundância, para ambas as localidades.

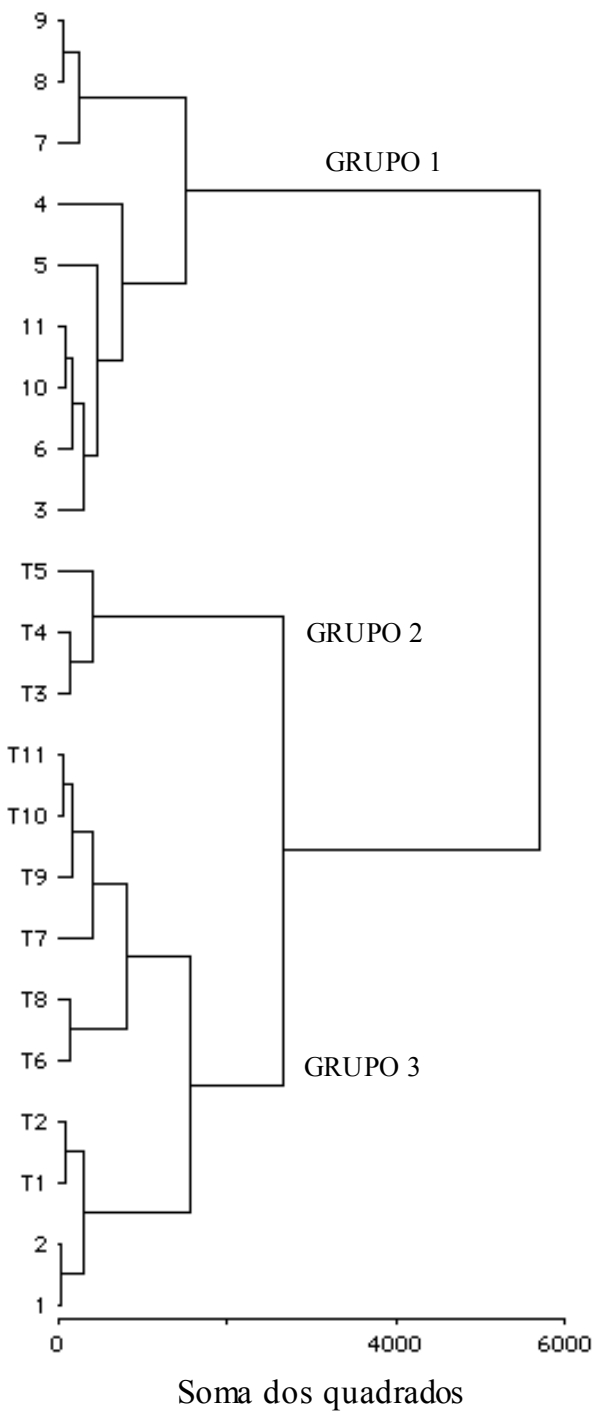

Figura 15. Dendograma das 22 unidades amostrais mostrando a estrutura dos grupos a partir da Análise de Agrupamentos pelo método da soma dos quadrados (variância mínima) usando a Distância Euclidiana. Os números de 1 a 11 correspondem aos meses de coleta (1: setembro, 2: outubro, 3:...) e a letra T referese as unidades amostrais do Arroio de Taquara, Rio Grande do Sul, os números sem letra referem-se ao Arroio de Tainhas. Os grupos formados após o teste de nitidez estão indicados.

Através do teste de Mantel observou-se que não houve correlação entre a abundância dos organismos e as variáveis abióticas.

\section{DISCUSSÃO}

A amplitude de variação da temperatura e da condutividade da água, caracteriza os arroios estudados. Taquara é um curso d'água típico de planície, com temperaturas mais amenas

Revista Brasileira de Zoologia 20 (1): 115-125, março 2003 
EIXO2 (15\%)

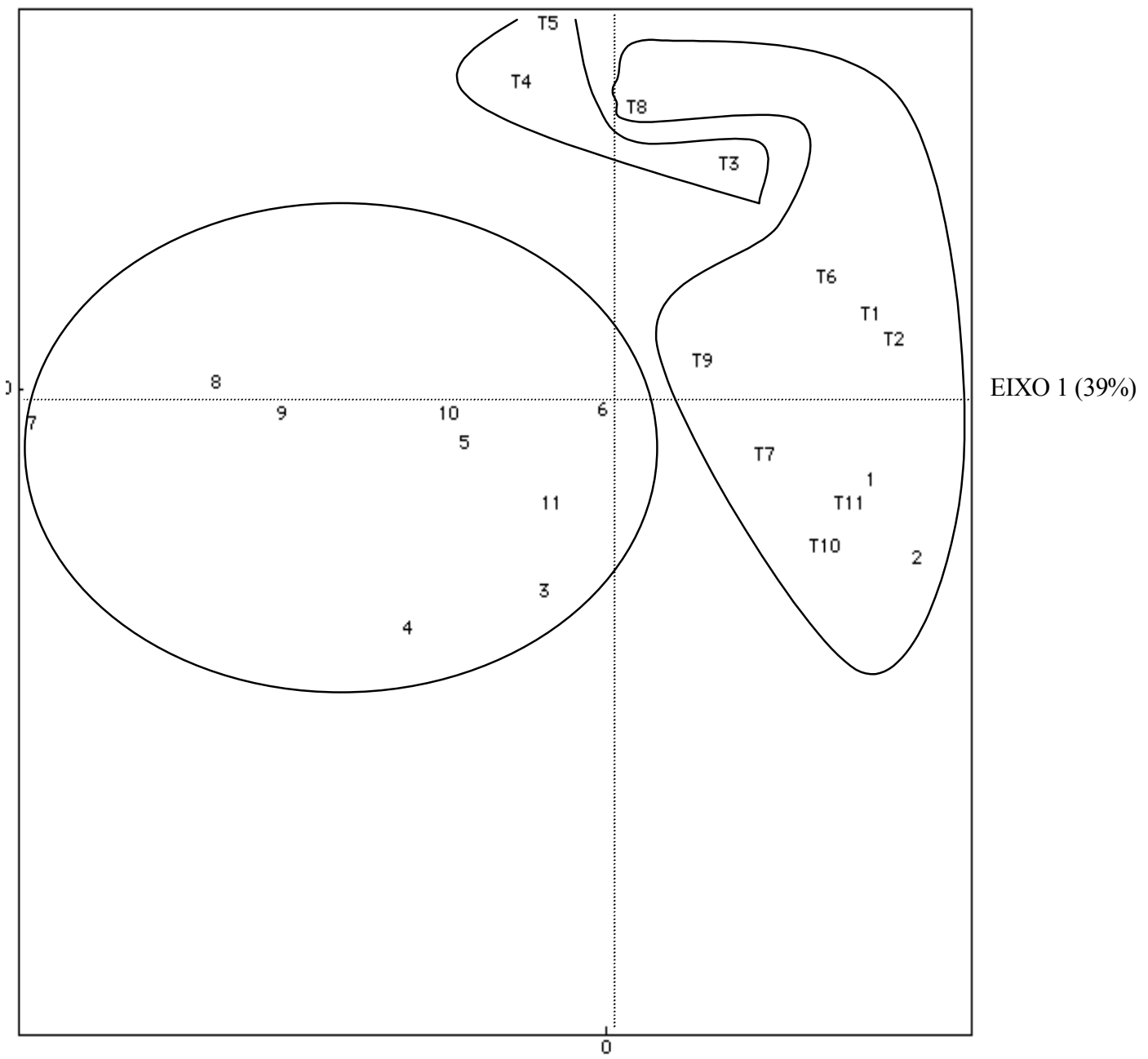

Figura 16. Diagrama de dispersão das 22 unidades amostrais nos eixos 1 e 2, obtidos por Análise de Coordenadas Principais com base em Distâncias Euclidianas, com os dados da composição faunística. Os números de 1 a 11 correspondem aos meses de coleta (1: setembro, 2: outubro, 3:...) e a letra T refere-se as unidades amostrais do Arroio de Taquara, Rio Grande do Sul, os números sem letra referem-se ao Arroio de Tainhas.

e com maior influência antrópica. Tainhas, que está localizado em uma região de planalto, com temperaturas e condutividades baixas, o ambiente sofre alguma influência somente do pastejo do gado e, portanto, mais preservado. Com base nos valores de temperatura e condutividade, Tainhas pode ser comparado a rios de primeira e segunda ordem, enquanto que Taquara a rios de sexta ordem, apesar dos locais amostrados serem típicos de rios de cabeceira.

Pesquisas realizadas em rios de primeira a sexta ordem verificaram que os locais com valores mais baixos de condutividade e alguns nutrientes pertenciam a cursos d'água de primeira e segunda ordem, enquanto que, aqueles com valores mais altos estavam entre os de quarta a sexta, locais degradados pela ação humana (BAPTISTA et al. 1998). A ação antrópica também foi observada nos córregos de São Paulo, onde as variações das características físicas e químicas são causadas pelos diversos impactos a que os rios são submetidos, como despejos de material orgânico e represamentos (GALLI \& Rocha 1998).

As pesquisas realizadas nos rios brasileiros não analisaram todos os nutrientes que foram utilizados no presente estudo, dificultando, portanto, a comparação com os dados. Entretanto, alguns autores verificaram as concentrações de alguns desses elementos. As concentrações de cálcio total e magnésio total das amostras de Tainhas são semelhantes àquelas encontradas em rios do Rio de Janeiro, como observado em BAPTISTA et al. $2001 \mathrm{~b}$. Os valores de fósforo total e sódio total foram bem mais

Revista Brasileira de Zoologia 20 (1): 115-125, março 2003 
elevados em Taquara. A presença destes nutrientes em altas concentrações é característica de ambiente eutrofizados, o que pode estar acontecendo neste local devido à presença de plantações de hortaliças nas margem do arroio (Allan 1995; Esteves 1998).

A maior abundância e riqueza de invertebrados em Tainhas pode ser devido ao maior grau de preservação do ambiente e, também, maior cobertura vegetal a montante do trecho amostrado, o que produz uma grande quantidade folhiço, o qual serve de alimento e abrigo para muitas larvas de insetos. $\mathrm{O}$ papel do folhiço como ponto de apoio para os insetos durante o período de seca, associado ao número de organismos por grama de folha, demonstra a importância das variáveis abióticas como o volume de água do rio, o substrato de fundo, a presença de mata ciliar e a velocidade da correnteza na distribuição dos invertebrados (UIEDA \& GaJARDO 1996).

A heterogeneidade do substrato rochoso, predominante em Tainhas, proporciona maior riqueza e abundância de espécies, enquanto que, em Taquara o substrato arenoso limita a distribuição de algumas famílias, devido à escassez de refúgio e disponibilidade de alimento. Este fato também foi observado em estudos realizados por Allan (1995), Beisel et al. (1998) e KiKUCH \& Uieda (1998). A natureza do substrato pode ser um fator limitante na composição da fauna. Algumas espécies apresentam adaptação as variações ambientais, como período de seca, principalmente com relação à sobrevivência de emigração, rápido ciclo de vida e sincronismo com o ciclo limnológico (Nessimian 1995a).

Com relação aos índices de diversidade, os dados de ambos arroios mostraram-se semelhantes aos encontrados em rios de regiões preservadas, como em Parques Ecológicos. Em córregos da região central do Brasil, os índices de diversidade foram calculados para três grupos de insetos, Ephemeroptera, Plecoptera e Trichoptera (EPT). O H' variou no período de seca e chuvoso, com valores de 1,00 a 3,58. Os índices mais baixos foram encontrados durante os meses de chuva, devido ao aumento do fluxo nos córregos o que acarreta um grande transporte de organismos rio abaixo (Oliveira et al. 1997; BISPO \& Oliveira 1998).

$\mathrm{Na}$ comparação entre dois locais de um mesmo rio em Lyon, na França, Charvet et al. (1998) encontraram diferenças significativas nos índices calculados, sendo a média de $\mathrm{H}^{\prime} 3,3$ no trecho superior do rio e 2,7 no inferior. Li et al. (2001) encontrou índices de Shannon-Wiener variando entre 1,40 a 3,31 em 16 rios do leste de Oregon, Estados Unidos. Um outro estudo, também nos EUA, obteve valores de $\mathrm{H}^{\prime}$ de 1,20 a 2,96 (SPONSElLer et al. 2001).

Apesar da diferença observada na temperatura e condutividade da água entre os arroios, verificou-se que os índices de diversidade encontrados em Tainhas e Taquara foram semelhantes. Estes resultados mostraram que os arroios possuem uma grande diversidade na fauna bentônica, apresentando valores dentro do esperado para rios de cabeceira, onde os níveis de preservação e o número de representantes da macrofauna são típicos destes locais.

A variação da abundância ao longo do ano pode estar associada às variáveis bióticas, como a reprodução. Na primavera, os índices foram mais baixos, provavelmente, devido à época reprodutiva dos adultos de muitas espécies de insetos. Como a maior parte dos organismos coletados estavam no estágio imaturo é de se esperar que eles sejam mais abundantes no período posterior à reprodução dos adultos, ou seja, no outono (Tainhas) e verão (Taquara). Resultados semelhantes, onde os macroinvertebrados foram mais freqüentes nos meses subseqüentes ao período reprodutivo, também foram observados por JACOBSEN et al (1997), JACOBSEN \& ENCALADA (1998), KiKUCHI \& Uieda (1998) e BAPTISTA et al. (2001a).

Devido ao fato das variáveis físicas e químicas serem similares entre os rios de corredeira, a macrofauna bentônica encontrada nos arroios estudados é bastante semelhante aquela observada por Friberg et al. (1997), BAPTISTA et al. (1998) e KIKUCHI \& Uieda (1998), Brittain et al. (2001), Lods-Crozet et al. (2001) onde também houve predominância de Diptera-Chironomidae. Diptera-Chironomidae quase sempre apresenta-se como dominante, tanto em ambientes lóticos como lênticos, devido a sua tolerância a situações extremas como hipóxia e grande capacidade competitiva (Nessimian 1995b; Marques et al. 1999; CALListo et al. 2001b).

As famílias Leptophlebiidae (Ephemeroptera) e Psephenidae (Coleoptera), que foram mais abundantes em Tainhas, são características de ambientes limpos, bem oxigenados e com correnteza de moderada a forte (Perez 1988). Os ácaros que foram bastante freqüentes neste local são importantes indicadores de qualidade da água (Thorp \& Covich 1991). Já as famílias Elmidae (Coleoptera) e Trichorythidae (Ephemeroptera), presentes em maior número em Taquara, são típicas de águas lênticas e pouco profundas (Perez 1988).

A grande quantidade de Ephemeroptera, Plecoptera e Trichoptera, avaliada pelo índice EPT, pode indicar ambientes ainda preservados, com uma fauna rica e diversa. Resultados obtidos em outros rios, como os pertencentes à Bacia do Rio Macaé, Rio de Janeiro (Brasil), os do sul da Inglaterra, os do leste de Oregon e sudeste da Virginia (USA), revelam a importância deste índice na avaliação da qualidade da água (WRight et al. 1993, NesSiMIAN 1997, BAPTISTA et al. 2001a, Li et al. 2001, SPONSELLER et al. 2001). No entanto, Callisto \& Esteves (1998) mencionam que, como a diversidade dos táxons nos rios brasileiros ainda é pouco conhecida, deve-se ter muita cautela ao interpretar os resultados obtidos pela classificação de grupos funcionais proposta por MERRIT \& Cummins (1984) para rios norte americanos.

A maior abundância de todos os grupos funcionais em Tainhas pode ser devido à grande disponibilidade de alimento fornecido pela vegetação ripária do local. Os coletores que foram os organismos mais freqüentes em ambos locais, também foram mais numerosos em rios de correnteza e de altitude e em lagoas da região serrana de Minas Gerais, Brasil (JaCobsen \& EnCALADA 1998, Marques et al. 1999, Rempel et al. 2000, CAllisto et al 2001a, GALDEAN et al 2001).

O amostrador Surber revelou não ser adequado para amostrar o crustáceo anomuro do gênero Aegla Leach, um macroinvertebrado bentônico predador muito abundante nos dois locais estudados (BOND-BUCKUP \& BUCKUP 1994). Por outro lado, o baixo número de predadores nas amostras obtidas no presente trabalho, comparados com a abundância dos demais organismos, pode ser um aspecto que favoreça o estabelecimento dessas comunidades e mereça uma investigação futura com vistas ao entendimento das relações interespecíficas.

Os resultados sobre abundância, riqueza e diversidade dos arroios de Taquara e Tainhas são informações pioneiras para os cursos d'água do Rio Grande do Sul, revelando características da ecologia e distribuição dos invertebrados bentônicos encontrados que podem subsidiar futuros monitoramentos 
ambientais nestas regiões. Assim, a preservação destes ambientes reveste-se de importância para o estabelecimento e manutenção de várias espécies de organismos bentônicos.

\section{AGRADECIMENTOS}

Ao Dr. Ludwig Buckup pelo auxílio nos trabalhos de campo. Ao CNPq pelas bolsas de Doutorado, de Produtividade em Pesquisa e de Iniciação Científica das autoras. Ao Programa de Pós-Graduação em Biologia Animal pelos auxílios concedidos para realização dos trabalhos de campo. Ao Sr Márcio Pons proprietário da fazenda Utopia II, onde realizaram-se as coletas do Arroio de Tainhas; ao Dr. Valério Pillar e doutorando Enio Egon Sosinski Jr., do Departamento de Ecologia, Instituto de Biociências, Universidade Federal do Rio Grande do Sul, pela realização das análises estatísticas; aos referees anônimos da Revista Brasileira de Zoologia que analisaram este manuscrito com importantes sugestões.

\section{REFERÊNCIAS BIBLIOGRÁFICAS}

AlLAN, J.D. 1995. Stream ecology: structure and function of running waters. Chapman \& Hall, London, 388p.

Baptista, D.F.; D.F. Buss; L.F.M. Dorvillé \& J.L. Nessiamian. 2001a. Diversity and habitat preference of aquatic insects along the longitudinal gradient of the Macaé River Basin, Rio de Janeiro, Brazil. Revista Brasileira de Biologia, Rio de Janeiro, 61 (2): 249-258.

Baptista, D.F.; L.F.M. Dorvillé; D.F. Buss \& J.L. Nessiamian. 2001b. Spatial and temporal organization of aquatic insects assemblages in the longitudinal gradient of a tropical river. Revista Brasileira de Biologia, Rio de Janeiro, 61 (2): 295304.

Baptista, D.F.; L.F.M. Dorvillé; D.F. Buss; J.L. Nessiamian \& L.H.J. SOARES. 1998. Distribuição de comunidades de insetos aquáticos no gradiente longitudinal de uma bacia fluvial do sudeste brasileiro. p. 191-207. In: J.L. Nessimian \& Carvalho, E. (Eds). Ecologia de insetos aquáticos. Rio de Janeiro, PPGE-UFRJ, Series Oecologia Brasiliensis, vol. 5, 309p.

Beisel, J.N.; P. Usseglio-Polatera.; S. Thomas. \& J.C. Moreteau. 1998. Stream community structure in relation to spatial variation: the influence of mesohabitat characteristics. Hydrobiologia, Dordrecht, 389: 73-88.

Bispo, P.C. \& L.G. Oliveira. 1998. Distribuição espacial de insetos aquáticos (Ephemeroptera, Plecoptera e Trichoptera) em córregos de cerrado do Parque Ecológico de Goiânia, Estado de Goiás, p. 175-189. In: J.L. Nessimian \& A.L. Carvalho (Eds.). Ecologia de Insetos Aquáticos. Rio de Janeiro, PPGEUFRJ, Series Oecologia Brasiliensis, vol. 5, 309p.

Bond-Buckup, G. \& L. Buckup. 1994. A família Aeglidae (Crustacea, Decapoda, Anomura). Arquivos de Zoologia, São Paulo, 2 (4): 159-346.

Borror, D.J. \& D.M. DeLong. 1969. Estudos dos insetos. São Paulo, Edgard e Blücher Ltda., 653p.

Branco, L.H.Z. \& O. Necchi Jr. 1997. Variação longituninal de parâmetros físicos e químicos em três rios pertencentes a diferentes bacias de drenagem na região noroeste do Estado de São Paulo. Acta Limnologica Brasiliensia, São Carlos, 9: 165-177.

Brittain, J.E.; S.J. Salveit; E. Castella; J. Bogen; T.E. Bonsnes.; I.
Blakar.; T. Bremnes; I. Haug. \& G. Velle. 2001. The macroinvertebrate communities of two contrasting Norwegian glacial rivers in relation to environmental variables. Freshwater Biology, Oxford, 46: 1723-1736.

Callisto, M. \& F.A. Esteves. 1998. Categorização funcional dos macroinvertebrados bentônicos em quatro ecossistemas lóticos sob influência das atividades de uma mineração de bauxita na Amazônia central (Brasil), p. 223-234. In: J.L. Nessimian \& A.L. Carvalho (Eds). Rio de Janeiro, PPGE-UFRJ, Series Oecologia Brasiliensis, vol. 5, 309p.

Callisto, M.; P. Moreno \& F.A.R. Barbosa. 2001a. Habitat diversity and benthic functional trophic groups at Serra do Cipó, Southeast Brazil. Revista Brasileira de Biologia, Rio de Janeiro, 61 (2): 259-266.

Callisto, M.; M. Moretti \& M. Goulart. 2001b. Macroinvertebrados bentônicos como ferramenta para avaliar a saúde de riachos. Revista Brasileira de Recursos Hídricos, Porto Alegre, 6 (1): 71-82.

Charvet, S.; A. Kosmala \& B. Statzner. 1998. Biomonitoring through biological traits of benthic macroinvertebrate: perspectives for a general tool in stream management. Archiv fur Hydrobiologie, Stuttgart, 142 (4): 415-432.

EDMONDSON, W.T. 1959. Freshwater biology. Jonh Wiley \& Sons, INC., New York, $2^{\text {nd }}$ ed., 1248p.

Esteves, F.A. 1998. Fundamentos de limnologia. Interciência $2^{\mathrm{a}}$ ed., Rio de Janeiro, 602p.

Friberg, N.; M.J. Winterbourn; K.A. Shearer \& S.E. Larsen. 1997. Benthic communities of forest streams in the South Island, New Zealand: effects of forest type and location. Archiv fur Hydrobiologie, Stuttgart, 138 (3): 289-306.

Galdean, N.; M. Callisto \& F.A.R. Barbosa. 2001. Biodiversity assessment of benthic macroinvertebrates in altitudinal lotic ecosystems of Serra do Cipó (MG, Brazil). Revista Brasileira de Biologia, Rio de Janeiro, 61 (2): 239-248.

Galli, S. \& O. RосHA. 1998. Aplicação da abordagem ecossistêmica ao estudo da microbacia dos corrégos Água da Prata e Macuco (São Carlos-SP). Anais do VIII Seminário Regional de Ecologia, São Carlos, 3: 1381-1387.

Ipagro. 1989. Atlas agroclimático do Estado do Rio Grande do Sul. Governo do Estado do Rio Grande do Sul, Secretaria da Agricultura e Abastecimento, Departamento de Pesquisa, Instituto de Pesquisas Agronômicas, Seção de Ecologia Agrícola, v.3, 211-326p.

JaCOBSEn, D. \& A. EnCALADA. 1998. The macroinvertebrate fauna of Ecuadorian highland streams in the wet and dry season. Archiv fur Hydrobiologie, Stuttgart, 142 (1): 53-70.

Jacobsen, D.; R. Schultz \& A. Encalada. 1997. Structure and diversity of stream invertebrate assemblages: the influence of temperature with altitude and latitude. Freshwater Biology, Oxford, 38: 247-261.

KIKUCHI, R.M. \& V.S. UIEDA. 1998. Composição da comunidade de invertebrados de um ambiente lótico tropical e sua variação espacial e temporal. pp. 157-173. In: J.L. Nessimian, \& E. Carvalho (Eds). Rio de Janeiro, PPGE-UFRJ, Series Oecologia Brasiliensis, vol. 5, 309p.

Krebs, C.J. 1989. Ecological methodology. New York, Copyright, 654p.

LI, J.; A. Herlihy; W. Gerth; P. Kaufmann; S. Gregory; S. Urquhart \& D.P. LARSEN. 2001. Variability in stream macroinvertebrate

Revista Brasileira de Zoologia 20 (1): 115-125, março 2003 
at multiple spatial scales. Freshwater Biology, Oxford, 46: 87-97.

Lods-Crozet, B.; E. Castella; D. Cambin; C. Ilg; S. Knispel \& H. MAYOR-SimEANT. 2001. Macroinvertebrate community structure in relation to environmental variables in a Swiss glacial stream. Freshwater Biology, Oxford, 46: 1641-1661.

MACAN, T.T. 1975. Guia de animales invertebrados de agua dulce. Pamplona, Eunsa, 118p.

MagurRan, A.E. 1988. Ecological diversity and its measurement. London, Croon Helm, 179p.

Mantel, N. 1967. The detection of disease clustering and a generalized regression approach. Cancer Research, Philadelphia, 27: 209-220.

Marques, M.G.S.M.; R.L. Ferreira \& F.A.R. Barbosa. 1999. A comunidade de macroinvertebrados aquáticos e características limnológicas das Lagoas Carioca e da Barra, Parque Estadual do Rio Doce, MG. Revista Brasileira de Biologia, Rio de Janeiro, 59 (2): 203-210.

Merritt, R.W. \& K.W. Cummins 1984. An introduction to the aquatic insects of North America. Dubuque, Kendall \& Hunt, $2^{\text {nd }}$ ed., 722p.

Nessimian, J.L. 1995a. Composição da fauna de invertebrados bentônicos em um brejo entre dunas no litoral do Estado do Rio de Janeiro, Brasil. Acta limnologica Brasiliensia, São Carlos, 7: 41-59.

. 1995b. Abundância e biomassa de macroinvertebrados de um brejo de dunas no litoral do Estado do Rio de Janeiro. Revista Brasileira de Biologia, Rio de Janeiro, 55 (4): 661-683.

. 1997. Categorização funcional de macroinvertebrados de um brejo de dunas no Estado do Rio de Janeiro. Revista Brasileira de Biologia, Rio de Janeiro, 57 (1): 135-145.

Nimer, E. 1989. Climatologia do Brasil. Rio de Janeiro, IBGE, $421 \mathrm{p}$.

Oliveira, L.G.; P.C. Bispo \& N.C. Ś́. 1997. Ecologia de comunidades de insetos bentônicos (Ephemeroptera, Plecoptera e Trichoptera), em córregos do Parque Ecológico de Goiânia, Goiás, Brasil. Revista Brasileira de Zoologia, Curitiba, 14 (4): 867-876.
Perez, G.R. 1988. Guía para el estudio de los macroinvertebrados acuáticos del Departamento de Antioquia. Fen Colombia, Colciencias, 217p.

Pillar, V.P. 1997. Multivariate exploratory analysis and randomization testing with MULTIV. Coenoses, Gorizias, 12: 145148.

1999a. The bootstrapped ordination re-examined. Journal of Vegetation Science, Uppsala, 10: 895-902. . 1999b. How sharp are classification? Ecology, Durham, 80 (8): 2508-2516.

Pillar, V.P. \& L. Orlóci. 1996. On randomization testing in vegetation science: multifactor comparisons of relevé groups. Journal of Vegetation Science, Uppsala, 7: 585-592.

Poole, R.W. 1974. An introduction to quantitative ecology. Tokyo, International Student Edition, 532p.

Rambo, B. 1956. A Fisionomia do Rio Grande do Sul. Porto Alegre, Edição Selbach \& Cia., vol. 6, 473p.

Rempel, L.L.; J. Richardson. \& M.C. Healey. 2000. Macroinvertebrate community structure along gradients of hydraulic and sedimentary conditions in a large gravel-bed river. Freshwater Biology, Oxford, 45: 57-73.

Schwoerbel, J. 1975. Métodos de hidrobiologia. Madrid, H. Blume ediciones, 262p.

Southwood, T.R.E. 1978. Ecology methods. London, Chapman $\&$ Hall, $2^{\text {nd }}$ ed., 524p.

Sponseller, R.A.; E.F. Benfield \& H.M. Valett. 2001. Relationships between land use, spatial scale and stream macroinvertebrate communities. Freshwater Biology, Oxford, 46: 1409-1424.

Thorp, J.H. \& A.P. Covich. 1991. Ecology and classification of North American freshwater invertebrates. San Diego, Academic press, Inc911p.

UIEDA, V.S. \& I.C.S.M. GAJARDO. 1996. Macroinvertebrados perifíticos encontrados em poções e corredeiras de um riacho. Naturalia, São Paulo, 21: 31-47.

Wright, J.F.; M.T. Furse; P.D. Armitage \& D. Moss. 1993. New procedures for identifying running-water sites subjects to environmental stress and for evaluating sites for conservation, based on the macroinvertebrate fauna. Archiv fur Hydrobiologie, Stuttgart, 127 (3): 319-326.

Recebido em 26.VIII.2002; aceito em 11.III.2003. 